\title{
The Mordell integral, quantum modular forms, and mock Jacobi forms
}

\author{
Bobbie Chern ${ }^{1 *}$ and Robert C Rhoades ${ }^{2}$
}

*Correspondence:
bgchern@stanford.edu
${ }^{1}$ Department of Electrical
Engineering, Stanford University,
Stanford, CA 94305, USA
Full list of author information is
available at the end of the article

\author{
Abstract \\ It is explained how the Mordell integral$$
\int_{\mathbb{R}} \frac{e^{\pi i \tau x^{2}-2 \pi z x}}{\cosh (\pi x)} d x
$$

unifies the mock theta functions, partial (or false) theta functions, and some of Zagier's quantum modular forms. As an application, we exploit the connections between $q$-hypergeometric series and mock and partial theta functions to obtain finite evaluations of the Mordell integral for rational choices of $\tau$ and $z$.

Mathematics Subject Classification: 11P55, $05 \mathrm{~A} 17$

Keywords: Jacobi forms; mock Jacobi Form; partial Jacobi theta function; False theta function; Partial theta function; Mock theta function; Mordell integral; Ramanujan

\section{The Mordell integral}

The Mordell integral is

$$
h(z ; \tau):=\int_{\mathbb{R}} \frac{e^{\pi i \tau w^{2}-2 \pi z w}}{\cosh (\pi w)} d w=2 \int_{0}^{\infty} e^{\pi i \tau w^{2}} \frac{\cosh (2 \pi z w)}{\cosh (\pi w)} d w .
$$

The integral was studied by Kronecker [19,20] and Lerch [23-25] in the late 1800s. A few years later, the integral underwent an intensive investigation by Mordell [28,29]. He established relationships between this integral and class number formulas. Later, Siegel [37] used properties of the integral to determine the approximate functional equation of the Riemman zeta function as well as asymptotics for its first moment. Very recently, the Mordell integral has been used to efficiently compute values of the Riemann zeta function [21]. The integral also appears in the solution of the 1-dimensional heat equation, see [32] and the references therein. Andrews [1] gave an early investigation of the Mordell integral in the study of the mock theta functions of Ramanujan.

The Mordell integral plays a central role in Sander Zwegers Ph.D. thesis [42] on the mock theta functions. His work shows that the Mordell integral may be viewed as the obstruction to modularity of the mock theta functions. Moreover, he showed how to reinterpret the integral in terms of a period integral of a certain weight $3 / 2$ modular form. His thesis has paved the way for many applications of mock theta functions to the study

C 2015 Chern and Rhoades; licensee Springer. This is an Open Access article distributed under the terms of the Creative 
of partitions and applications to black holes and moonshine (see [9] and [7,8,11,12]), for example. See the survey of Ono [31] and the Bourbaki lecture of Zagier [39] for details.

The most significant applications of mock theta functions to the theory of partitions come from the study of Dyson's rank (see the works of Bringmann and Ono [4,5]). An integer partition of $n$ is a sequence $\lambda_{1} \geq \lambda_{2} \geq \cdots \geq \lambda_{\ell}>0$ such that $\lambda_{1}+\lambda_{2}+\cdots+\lambda_{\ell}=n$. The numbers $\lambda_{j}$ are the parts of the partition. Dyson's rank [10] of a partition is the largest part minus the number of parts. For example, $5 \geq 4 \geq 4 \geq 3 \geq 3 \geq 2 \geq 1 \geq 1 \geq 1 \geq 1$ is a partition of 25 with rank $5-10=-5$. Let $N(m, n)$ denote the number of integer partitions of $n$ with rank $m$. Define

$$
R(u ; \tau):=1+\sum_{n=1}^{\infty} \sum_{m=-\infty}^{\infty} N(m, n) w^{m} q^{n}=\sum_{n=0}^{\infty} \frac{q^{n^{2}}}{(w q ; q)_{n}\left(w^{-1} q ; q\right)_{n}},
$$

where $w=e^{2 \pi i u}, q=e^{2 \pi i \tau}$ with $\tau \in \mathbb{H}=\{x+i y: x, y \in \mathbb{R}, y>0\},(a ; q)_{n}:=$ $\prod_{j=0}^{n-1}\left(1-a q^{j}\right)$ for positive integers $n$, and $(a ; q)_{0}:=1$. Zwegers's thesis [42] shows that when $z \in \mathbb{Q}+\tau \mathbb{Q}$, the function $R(z ; \tau)$ may be viewed as a mock theta function (see [39]).

The next theorem shows that the partial theta function

$$
\psi(u ; \tau):=\sum_{n=0}^{\infty}\left(\frac{12}{n}\right) e^{-\pi i n u} q^{-\frac{n^{2}}{24}}
$$

defined for $\tau \in \mathbb{H}^{-}$, has a modular transformation property that mirrors that for $R(u ; \tau)$. Here, $(\vdots)$ is the Kronecker symbol. The function is a "partial theta function" because it is the sum over half of the integer lattice.

Theorem 1.1. Define $\widetilde{R}(u ; \tau)=\frac{i q \frac{1}{24}}{2 \sin (\pi u)} R(u ; \tau)$. For $\tau \in \mathbb{H}=\{x+i y: x, y \in \mathbb{R}, y>0\}$ and $u \in \mathbb{C} \backslash(\mathbb{Z}+\tau \mathbb{Z})$

$$
\widetilde{R}(u ; \tau)-\frac{e^{\frac{3 \pi i u^{2}}{\tau}}}{\sqrt{i \tau}} \widetilde{R}\left(\frac{u}{\tau} ;-\frac{1}{\tau}\right)=\frac{1}{2} q^{-\frac{1}{6}} \sum_{ \pm} \pm e^{\mp 2 \pi i u} h(3 u \pm \tau ; 3 \tau) .
$$

On the other hand, for $\tau \in\left(0, \frac{1}{2}\right)$ and $u \in \mathbb{H}^{-}=\{x+i y: x, y \in \mathbb{R}, y<0\}$ and $\operatorname{Re}(u)<\frac{1}{6}$

$$
\psi(u ; \tau)-\frac{e^{\frac{3 \pi i u^{2}}{\tau}}}{\sqrt{i \tau}} \psi\left(\frac{u}{\tau} ;-\frac{1}{\tau}\right)=\frac{1}{2} q^{-\frac{1}{6}} \sum_{ \pm} \pm e^{\mp 2 \pi i u} h(3 u \pm \tau ; 3 \tau) .
$$

Remarks. (1). The first part of Theorem 1.1 is deduced from the work of Zwegers [42]. The second part uses a result of Ramanujan [34]. These results can be combined to give a similar theorem for any family of mock theta functions such as $R(u ; \tau)$. In particular, similar theorems exist for the universal mock theta functions $g_{2}(u ; \tau)$ and $g_{3}(u ; \tau)$ defined by Gordon and McIntosh [16].

(2). Theorem 1.1 shows that mock theta function $\widetilde{R}$ and partial theta function $\psi$, together, provide a function which is defined on $\mathbb{H} \cup \mathbb{H}^{-}$and has a pseudo-modular property which leaks from $\mathbb{H}$ onto $\mathbb{R}$. Moreover, if we interpret the function at rationals in terms of their radial limits (when they exist), then we obtain quantum modular forms (see Section 1.1).

(3). Often partial theta functions may be written as false theta functions. A partial theta function is half of a theta series (for instance the sum is over the positive integers), while a false theta function is a theta series with some of the signs incorrect. Indeed, this is 
true for many of Rogers original examples of false theta functions. For example, it is not difficult to see that

$$
\sum_{n \geq 0}\left(\frac{-12}{n}\right) q^{\frac{n^{2}-1}{24}}=\sum_{n \geq 0} q^{\frac{n(3 n+1)}{2}}\left(1-q^{2 n+1}\right)=\sum_{n \geq 0} q^{\frac{n(3 n+1)}{2}}-\sum_{n \leq-1} q^{\frac{n(3 n+1)}{2}} .
$$

Hence, Theorem 1.1 shows that the False $\vartheta$-functions and 'Mock' $\vartheta$-functions are two sides of the same coin. This is contrary to Ramanujan's claim in his deathbed letter that, "Unlike the False $\vartheta$-functions (studied partially by Prof. Rogers in his interesting paper) they ('Mock' $\vartheta$-functions) enter into mathematics as beautifully as the ordinary theta functions."

(4). The connection between partial and mock theta functions in different half-planes has appeared in at least three other contexts. (A) Results of Lawerence-Zagier [22] and Hikami [18] show how the asymptotics of each object toward rationals are related. (B) The second author [36] showed that Rademacher's "expansions of zero" phenomenon [33] extends from the theory of classical modular forms to the theory of mock theta functions. In this approach, one sees that the relevant Poincaré series yield a mock theta function in one half-plane and a partial theta function in the complementary halfplane. (C) Zwegers $[22,41]$ has given a number of examples of $q$-hypergeometric series which equal mock theta functions in one domain and partial theta functions in a disjoint domain. Indeed, Theorem 1.4, below, provides an infinite family of such examples. The role of $q$-hypergeometric series in this connection has been studied extensively by the second author in works with Bringmann and Folsom [3] and Li and Ngo [26]. A similar relationship was also found by Mortenson with Hickerson [17,30].

The next section discusses the connection between the Mordell integral and Zagier's quantum modular forms. As an application we give evaluations of special values of the Mordell integral in terms of $q$-hypergeometric series.

\subsection{Zagier's quantum modular forms}

In a 1997 Max Planck lecture Zagier credits Kontsevich with introducing the function

$$
F(q):=\sum_{n=0}^{\infty}(q ; q)_{n}=1+(1-q)+(1-q)\left(1-q^{2}\right)+(1-q)\left(1-q^{2}\right)\left(1-q^{3}\right)+\ldots
$$

where $(x ; q)_{n}:=\prod_{j=0}^{n-1}\left(1-x q^{j}\right)$. This function, called "Kontsevich's strange function", exists only when $q$ is a root of unity. With $\zeta_{k}:=e^{2 \pi i / k}$, Kontsevich conjectured the following elegant asymptotic expansion

$$
F\left(\zeta_{k}\right) \sim e^{-\frac{\pi i}{12}\left(k-3+\frac{1}{k}\right)} k^{\frac{3}{2}}+\sum_{n=0}^{\infty} \frac{b_{n}}{n !}\left(-\frac{2 \pi i}{k}\right)^{n} \text { as } k \rightarrow \infty
$$

for some constants $b_{n}$. Zagier [38] proved this asymptotic, with explicit $b_{n}$. Moreover, he proved that this function satisfies the "identity"

$$
F(q)=-\frac{1}{2} \sum_{n=1}^{\infty} n\left(\frac{12}{n}\right) q^{\frac{n^{2}-1}{24}} .
$$

Neither side of this identity makes sense simultaneously. Indeed, the right hand side converges in the unit disk $|q|<1$, but nowhere on the unit circle. The identity means that $F(q)$ at roots of unity agrees with the radial limit of the right hand side. 
With this example, and a few others in mind, Zagier [40] introduced the notion of a quantum modular form. A weight $k$ quantum modular form is a complex-valued function $f$ on $\mathbb{Q}$, or possibly $\mathbb{P}^{1}(\mathbb{Q}) \backslash S$ for some set $S$, such that for all $\gamma=\left(\begin{array}{ll}a & b \\ c & d\end{array}\right) \in \mathrm{SL}_{2}(\mathbb{Z})$ the function

$$
h_{\gamma}(x):=f(x)-\epsilon(\gamma)(c x+d)^{-k} f\left(\frac{a x+b}{c x+d}\right)
$$

satisfies a "suitable" property of continuity or analyticity. The $\epsilon(\gamma)$ are suitable complex numbers, such as those in the theory of half-integral weight modular forms when $k \in$ $\frac{1}{2} \mathbb{Z} \backslash \mathbb{Z}$.

In this paper we demonstrate how the study of the Mordell integral yields asymptotics such as (1.5) and identities such as (1.6).

Consider the second order mock theta function of McIntosh [27] defined for $|q|<1$ by

$$
B\left(q^{\frac{1}{2}}\right):=\sum_{n=0}^{\infty} \frac{q^{\frac{n(n+1)}{2}}(-q ; q)_{n}}{\left(q^{\frac{1}{2}} ; q\right)_{n+1}^{2}} .
$$

For certain roots of unity $q$, the series defining $B\left(q^{\frac{1}{2}}\right)$ will converge. For example,

$$
B(-1)=\frac{1}{2} \text { and } B\left(\zeta_{6}\right)=-0.25+1.2990381056766 i \text {. }
$$

Data suggests an asymptotic analogous to (1.5) for $B\left(\zeta_{2 k}\right)$ (see Figure 1). We have the following theorem.

Theorem 1.2. For each odd $k \geq 1, B\left(\zeta_{2 k}\right) \in \mathbb{Q}\left(\zeta_{2 k}\right)$. Moreover, we have

$$
\begin{aligned}
-\zeta_{4 k}^{-1} \cdot B\left(\zeta_{2 k}\right) & =-\sqrt{\frac{k i}{2}} \zeta_{16}^{k}+\sqrt{\frac{k i}{2}} \int_{0}^{\infty} \frac{e^{-\frac{\pi i k}{2} w^{2}}}{\cosh (\pi w)} d w \\
& \sim-\zeta_{16}^{k} \sqrt{\frac{k i}{2}}+\frac{1}{2} \sum_{n=0}^{\infty} \frac{(-1)^{n} E_{2 n}}{n !}\left(\frac{\pi}{2 i k}\right)^{n} \text { as } k \rightarrow \infty \text { with } k \text { odd }
\end{aligned}
$$

where $E_{n}$ are the Euler numbers.

Moreover, we have the following result which is the analog of (1.6).

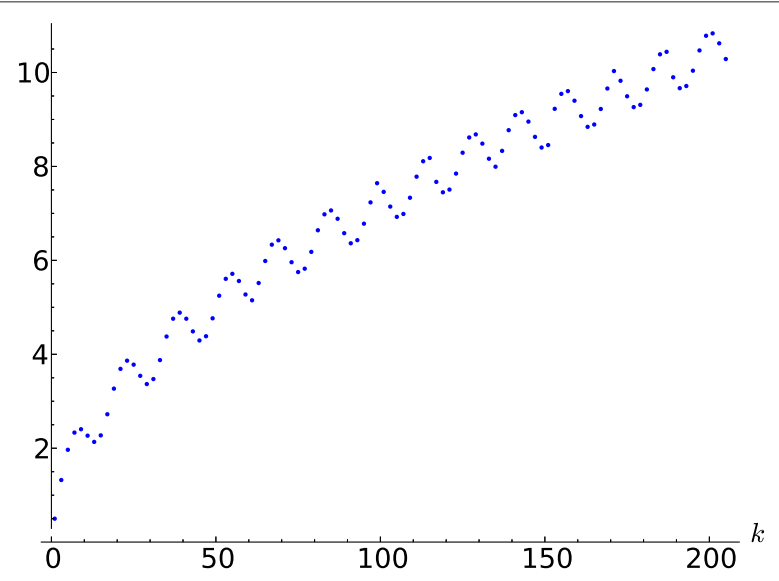

Figure $1 \mathrm{~A}$ plot of $\left|B\left(\zeta_{2 k}\right)\right|$ for odd values of $k$. 
Proposition 1.3. Let

$$
b(q):=\sum_{n=0}^{\infty}(-1)^{n} q^{n(n+1)}
$$

For any root of unity $\zeta_{2 k}$ we have

$$
B\left(\zeta_{2 k}\right)=-b\left(\zeta_{k}^{-1}\right)
$$

where the identity makes sense as a radial limit.

The mock theta function $B(q)$ fits into a natural two parameter family of mock theta functions. To state the theorem we introduce two families of $q$-hypergeometric series which are given as specializations of the Rogers-Fine series

$$
F(a, b ; t: q):=\sum_{n=0}^{\infty} \frac{(a q ; q)_{n}}{(b q ; q)_{n}} t^{n} .
$$

Here we consider the following specializations

$$
\begin{aligned}
& G(a, b ; \tau):=\frac{q^{\frac{a^{2}}{b^{2}}}}{1-q^{\frac{a}{b}}} \cdot F\left(-q^{\frac{a}{b}-1}, q^{\frac{a}{b}} ;-q^{\frac{a}{b}}: q\right), \\
& H(a, b ; \tau):=q^{\frac{1}{8}} \cdot F\left(\zeta_{b}^{-a} q^{-1}, \zeta_{b}^{-a} ; \zeta_{b}^{-a} q: q^{2}\right) .
\end{aligned}
$$

As explained in Theorem 1.3 of [15] these series define functions for $\tau \in \mathbb{H} \cup \mathbb{H}^{-}$and certain $\tau \in \mathbb{Q}$.

Theorem 1.4. The following are true.

(A) For $\tau \in \mathbb{H}^{-}$these series are mock theta functions according to Zagier's definition [39].

(B) For $\tau \in \mathbb{H}$ these series may be written as partial theta functions. We have

$$
\begin{aligned}
& G(a, b ; \tau)=\sum_{n=0}^{\infty}(-1)^{n} q^{\left(n+\frac{a}{b}\right)^{2}} \\
& H(a, b ; \tau)=\sum_{n=0}^{\infty} \zeta_{b}^{-a n} q^{\frac{1}{2}\left(n+\frac{1}{2}\right)^{2}}
\end{aligned}
$$

(C) Let $0<a<b$ be coprime integers, with $b$ even, and let

$$
Q_{a, b}:=\left\{\frac{h}{k} \in \mathbb{Q}: \operatorname{gcd}(h, k)=1, h>0, b \mid 2 h, b \nmid h, k \equiv a(\bmod b), k \geq a\right\} .
$$

For $x \in Q_{a, b} \cap(0,1)$, we have that

$$
\begin{aligned}
\frac{1}{2} h\left(\frac{a}{b}-\frac{1}{2} ;-\frac{1}{2 x}\right) & =\int_{0}^{\infty} e^{-\frac{\pi i}{2 x} w^{2}} \frac{\cosh \left(\left(2 \frac{a}{b}-1\right) \pi w\right)}{\cosh (\pi w)} d w \\
& =\sqrt{-2 i x} G(a, b ;-x)+i e^{-\frac{\pi i a}{b}} \cdot H\left(a, b ; \frac{1}{2 x}\right) .
\end{aligned}
$$

Moreover, the sums defining $G(a, b ;-x)$ and $H(a, b ; 1 / 2 x)$ are finite for these $x$. 
For $a=1$ and $b=2$ this theorem yields the values of the mock theta function $B\left(q^{\frac{1}{2}}\right)$. In particular, a well-known identity for $B\left(q^{\frac{1}{2}}\right)$, see [16], gives for $|q|<1$

$$
B\left(q^{\frac{1}{2}}\right)=\sum_{n=0}^{\infty} \frac{q^{\frac{n}{2}}\left(-q^{\frac{1}{2}} ; q\right)_{n}}{\left(q^{\frac{1}{2}} ; q\right)_{n+1}}
$$

Thus, we have

$$
G\left(1,2 ;-\frac{1}{k}\right)=\zeta_{4 k}^{-1} \sum_{n=0}^{\frac{k-1}{2}}(-1)^{n} \zeta_{2 k}^{-n} \frac{\left(-\zeta_{2 k}^{-1} ; \zeta_{k}^{-1}\right)_{n}}{\left(\zeta_{2 k}^{-1} ; \zeta_{k}^{-1}\right)_{n+1}}=-\zeta_{4 k}^{-1} B\left(\zeta_{2 k}\right)
$$

and $H\left(1,2 ; \frac{k}{2}\right)=\zeta_{16}^{k}$. Combining these calculations and using Theorem 1.4 (D) we find, after complex conjugation, that

$$
\frac{1}{2} h\left(0 ; \frac{k}{2}\right)=\int_{0}^{\infty} \frac{e^{\frac{\pi i k}{2} w^{2}}}{\cosh (\pi w)} d w=\zeta_{16}^{-k}+\sqrt{\frac{2 i}{k}} \zeta_{4 k} \sum_{n=0}^{\frac{k-1}{2}}(-1)^{n} \zeta_{2 k}^{n} \frac{\left(-\zeta_{2 k} ; \zeta_{k}\right)_{n}}{\left(\zeta_{2 k} ; \zeta_{k}\right)_{n+1}} .
$$

This is the first part of Theorem 1.2. Additionally, Proposition 1.3 follows from Theorem 1.4 (A) and (B).

In Sections 2 and 3 we prove Theorem 1.1. In Section 4 we give the proofs of Theorem 1.4 and Theorem 1.2.

\section{The Mordell Integral after Ramanujan}

In this section the Mordell integral is represented in terms of the partial theta function. The result is used to deduce the second part of Theorem 1.1. The following result, slightly reworded, appears in Ramanujan's article [34]. In particular, see (19) and (20) on page 62 of [34].

Theorem 2.1. If $\tau$ is a positive real number and $z \in \mathbb{H}^{-}=\{x+i y: x, y \in \mathbb{R}, y<0\}$ with $|\operatorname{Re}(z)|<\frac{1}{2}$, we have

$$
\frac{1}{2} h(z ; \tau)=\eta(z ; \tau)+e^{\frac{\pi i z^{2}}{\tau}} \sqrt{\frac{i}{\tau}} \eta\left(\frac{z}{\tau} ;-\frac{1}{\tau}\right)
$$

where

$$
\eta(z ; \tau):=\sum_{n=0}^{\infty}\left(\frac{-4}{n}\right) e^{-\pi i n z} e^{-\pi i \tau \frac{n^{2}}{4}} .
$$

of second part of Theorem 1.1.

We consider

$$
\frac{1}{2} \sum_{ \pm} \pm e^{\mp 2 \pi i u} h(3 u \pm \tau ; 3 \tau)=\sum_{ \pm} \pm e^{\mp 2 \pi i u} \eta(3 u \pm \tau ; 3 \tau)+\frac{\sqrt{i e^{\frac{3 \pi i u^{2}}{\tau}}} q^{\frac{1}{6}}}{\sqrt{3 \tau}} \sum_{ \pm} \pm \eta\left(\frac{u}{\tau} \pm \frac{1}{3} ;-\frac{1}{3 \tau}\right) .
$$


First, we have

$$
\begin{aligned}
\sum_{ \pm} & \pm e^{\mp 2 \pi i u} \eta(3 u \pm \tau ; 3 \tau) \\
& =e^{-2 \pi i u} \sum_{n=0}^{\infty}\left(\frac{-4}{n}\right) e^{-3 \pi i n u-\pi i n \tau-\frac{3 \pi i n^{2} \tau}{4}}-e^{2 \pi i u} \sum_{n=0}^{\infty}\left(\frac{-4}{n}\right) e^{-3 \pi i n u+\pi i n \tau-\frac{3 \pi i n^{2} \tau}{4}} \\
& =\sum_{n=0}^{\infty}\left(\frac{-4}{n}\right) e^{-\pi i(3 n+2)-\frac{\pi i \tau}{12}\left((3 n)^{2}+12 n\right)}-\sum_{n=0}^{\infty}\left(\frac{-4}{n}\right) e^{-\pi i(3 n-2)-\frac{\pi i \tau}{12}\left((3 n)^{2}-12 n\right)} \\
& =-q^{\frac{1}{6}} \sum_{n=0}^{\infty} \chi(n) e^{-\pi i u n} q^{-\frac{n^{2}}{24}} \\
& =-q^{\frac{1}{6}} \psi(u ; \tau)
\end{aligned}
$$

where

$$
\chi(n):=\left\{\begin{array}{llll}
-1 & n \equiv 2 & (\bmod 3) \text { and } \frac{n-2}{3} \equiv 1 & (\bmod 4) \\
-1 & n \equiv 1 & (\bmod 3) \text { and } \frac{n+2}{3} \equiv 3 & (\bmod 4) \\
1 & n \equiv 2 & (\bmod 3) \text { and } \frac{n-2}{3} \equiv 3 & (\bmod 4) \\
1 & n \equiv 1 & (\bmod 3) \text { and } \frac{n+2}{3} \equiv 1 & (\bmod 4) \\
0 & \text { else } & &
\end{array}\right.
$$

The final line follows from $\chi(n)=\left(\frac{12}{n}\right)$.

On the other hand

$$
\begin{aligned}
\sum_{ \pm} \eta\left(\frac{u}{\tau} \pm \frac{1}{3} ;-\frac{1}{3 \tau}\right) & =\sum_{n=0}^{\infty}\left(\frac{-4}{n}\right) e^{-\pi i n \frac{u}{\tau}+\frac{\pi i n^{2}}{12 \tau}} \sum_{ \pm} \pm e^{\mp \frac{\pi i n}{3}} \\
& =-2 i \sum_{n=0}^{\infty}\left(\frac{-4}{n}\right) \sin \left(\frac{\pi n}{3}\right) e^{-\pi i n \frac{u}{\tau}} q_{1}^{-\frac{n^{2}}{24}} \\
& =-\sqrt{3} i \sum_{n=0}^{\infty}\left(\frac{12}{n}\right) e^{-\pi i n \frac{u}{\tau}} q_{1}^{-\frac{n^{2}}{24}} \\
& =-i \sqrt{3} \psi\left(\frac{u}{\tau} ;-\frac{1}{\tau}\right)
\end{aligned}
$$

since $\left(-\frac{4}{n}\right) \sin \left(\frac{\pi n}{3}\right)=\frac{\sqrt{3}}{2}\left(\frac{12}{n}\right)$. Hence

$$
\frac{1}{2} \sum_{ \pm} \pm e^{\mp 2 \pi i u} h(3 u \pm \tau ; 3 \tau)=-q^{\frac{1}{6}}\left(\psi(u ; \tau)-\frac{e^{\frac{3 \pi i u^{2}}{\tau}}}{\sqrt{i \tau}} \psi\left(\frac{u}{\tau} ;-\frac{1}{\tau}\right)\right) .
$$

Rewriting the sum of Mordell integrals gives the second claim of the theorem.

\section{The Mordell integral after Zwegers}

In this section we will prove the first part of Theorem 1.1. The result follows from Zwegers's paper [43] (which can be derived from his thesis [42]).

Define

$$
A_{\ell}(u, v ; \tau):=a^{\frac{\ell}{2}} \sum_{n \in \mathbb{Z}} \frac{(-1)^{\ell n} q^{\frac{\ell n(n+1)}{2}} b^{n}}{1-a q^{n}}
$$


where $a=e(u), b=e(v), q=e(\tau)$, and $e(x):=e^{2 \pi i x}$. Moreover, define

$$
\widehat{A}_{\ell}(u, v ; \tau)=A_{\ell}(u, v ; \tau)+\frac{i}{2} \sum_{k=0}^{\ell-1} e^{2 \pi i k u} \vartheta(v+k \tau+(\ell-1) / 2 ; \ell \tau) S(\ell u-v-k \tau-(\ell-1) / 2 ; \ell \tau),
$$

where

$$
\begin{aligned}
\vartheta(v ; \tau) & =\sum_{n \in \mathbb{Z}} e^{2 \pi i\left(n+\frac{1}{2}\right)\left(\nu+\frac{1}{2}\right)+\pi i(2 n+1) \frac{\tau}{4}} \\
& =-i e^{\frac{\pi i \tau}{4}-\pi i v} \prod_{n=1}^{\infty}\left(1-e^{2 \pi i n \tau}\right)\left(1-e^{2 \pi i v+2 \pi i(n-1) \tau}\right)\left(1-e^{-2 \pi i \nu+2 \pi i n \tau}\right)
\end{aligned}
$$

is the Jacobi theta function and

$$
S(u ; \tau):=\sum_{v \in \frac{1}{2}+\mathbb{Z}}(\operatorname{sgn}(v)-E((v+\operatorname{Im}(u) / \operatorname{Im}(\tau)) \sqrt{2 \operatorname{Im}(\tau)}))(-1)^{\nu-\frac{1}{2}} q^{-v^{2} / 2} e^{-2 \pi i v u}
$$

with $u \in \mathbb{C}, \tau \in \mathbb{H}$ and

$$
\begin{aligned}
& E(z):=2 \int_{0}^{z} e^{-\pi u^{2}} d u=\operatorname{sgn}(z)\left(1-\beta\left(z^{2}\right)\right), \quad z \in \mathbb{R} \\
& \beta(x):=\int_{x}^{\infty} u^{-\frac{1}{2}} e^{-\pi u} d u, \quad x \in \mathbb{R}_{\geq 0} .
\end{aligned}
$$

We collect the following results concerning the function $S(u ; \tau)$.

Proposition 3.1 (Proposition 1.9 of [42]). The function $S$ has the following transformation properties

$$
\begin{aligned}
& \text { 1. } S(u+1 ; \tau)=-S(u ; \tau) \\
& \frac{1}{\sqrt{-i \tau}} e^{\frac{\pi i u^{2}}{\tau}} S\left(\frac{u}{\tau} ;-\frac{1}{\tau}\right)+S(u ; \tau)=h(u ; \tau) .
\end{aligned}
$$

Proposition 3.2 (Proposition 2.3 of [2]). For $n \in \mathbb{N}, u \in \mathbb{C}, \tau \in \mathbb{H}$ we have

$$
S\left(u ; \frac{\tau}{n}\right)=\sum_{\ell=0}^{n-1} q^{-\frac{1}{2 n}\left(\ell-\frac{n-1}{2}\right)^{2}} e^{-2 \pi i u\left(\ell-\frac{n-1}{2}\right)\left(u+\frac{1}{2}\right)} S\left(n u+\left(\ell-\frac{n-1}{2}\right) \tau+\frac{n-1}{2} ; n \tau\right) .
$$

We will also require the following properties of the Jaocbi theta function.

Proposition 3.3. For $a \in \mathbb{C}$ and $b \in \mathbb{H}$ we have

1. $\vartheta(-a ; b)=-\vartheta(a ; b)$

2. $\vartheta(a+1 ; b)=-\vartheta(a ; b)$.

3. $\vartheta(0 ; b)=0$.

4. $\vartheta\left(\frac{a}{b} ;-\frac{1}{b}\right)=-i \sqrt{-i b} e^{\frac{\pi i a^{2}}{b}} \vartheta(a ; b)$.

5. For $n \in \mathbb{N}$

$$
\vartheta\left(a ; \frac{b}{n}\right)=\sum_{\ell=0}^{n-1} q^{\left(\ell-\frac{n-1}{2}\right)^{2} \frac{1}{2 n}} e^{2 \pi i\left(\ell-\frac{n-1}{2}\right)\left(a+\frac{1}{2}\right)} \vartheta\left(n a+\left(\ell-\frac{n-1}{2}\right) b+\frac{n-1}{2} ; n b\right) .
$$


The first four claims can be found in Proposition 1.3 of [42] and the final claim can be found on page 10 of [43].

Theorem 3.4 (Zwegers [43]). In the notation above we have the following

1. $\widehat{A}_{\ell}(-u,-v ; \tau)=-\widehat{A}_{\ell}(u, v ; \tau)$.

2. For all $n_{1}, n_{2}, m_{1}, m_{2} \in \mathbb{Z}$ we have

$\widehat{A}_{\ell}\left(u+n_{1} \tau+m_{1}, v+n_{2} \tau+m_{2} ; \tau\right)=(-1)^{\ell\left(n_{1}+m_{1}\right)} a^{\ell n_{1}-n_{2}} b^{-n_{1}} q^{\ell n_{1}^{2} / 2-n_{1} n_{2}} \widehat{A}_{\ell}(u, v ; \tau)$.

3. For all $\left(\begin{array}{ll}a & b \\ c & d\end{array}\right) \in S L_{2}(\mathbb{Z})$ we have

$$
\widehat{A}_{\ell}\left(\frac{u}{c \tau+d}, \frac{v}{c \tau+d} ; \frac{a \tau+b}{c \tau+d}\right)=(c \tau+d) e^{\pi i c\left(-\ell u^{2}+2 u v\right) /(c \tau+d)} \widehat{A}_{\ell}(u, v ; \tau) .
$$

If $R(u ; \tau)$ is the usual rank generating function defined in the introduction, then we have

$$
R(u ; \tau)=2 i a^{-1} q^{\frac{1}{24}} \sin (-\pi u) \frac{1}{\eta(\tau)} A_{3}(u,-\tau ; \tau) \text { and } \widetilde{R}(u ; \tau)=\frac{e^{-2 \pi i u}}{\eta(\tau)} A_{3}(u,-\tau ; \tau),
$$

where

$$
\eta(\tau):=q^{\frac{1}{24}} \prod_{n=1}^{\infty}\left(1-q^{n}\right)
$$

is the Dedekind eta-function (see, for instance, [39]).

Proof of the first part of Theorem 1.1. By Theorem 3.4

$$
\widehat{A}_{3}\left(\frac{u}{\tau},-\frac{\tau}{\tau} ;-\frac{1}{\tau}\right)=\tau e^{\pi i\left(-3 u^{2}-2 u \tau\right) / \tau} \widehat{A}_{3}(u,-\tau ; \tau) .
$$

By Proposition 3.3 (1)-(3) we have

$$
\begin{aligned}
\vartheta(-\tau+1 ; 3 \tau) & =-\vartheta(-\tau ; 3 \tau)=\vartheta(\tau ; 3 \tau), \\
\vartheta(-\tau+\tau+1 ; 3 \tau) & =\vartheta(1 ; 3 \tau)=-\vartheta(0 ; 3 \tau)=0, \\
\vartheta(-\tau+2 \tau+1 ; 3 \tau) & =-\vartheta(\tau ; 3 \tau) .
\end{aligned}
$$

Hence, using Proposition 3.1 (1) we have

$$
\widehat{A}_{3}(u,-\tau ; \tau)=A_{3}(u,-\tau ; \tau)-\frac{i}{2} e^{2 \pi i u} \vartheta(\tau ; 3 \tau) \sum_{ \pm} \pm e^{\mp 2 \pi i u} S(3 u \pm \tau ; 3 \tau)
$$

Proposition 3.1 (2) gives

$$
\pm e^{\mp 2 \pi i u} S(3 u \pm \tau ; 3 \tau)=\mp e^{\mp 2 \pi i u} \frac{e^{\pi i(3 u \pm \tau)^{2} /(3 \tau)}}{\sqrt{-3 i \tau}} S\left(\frac{u}{\tau} \pm \frac{1}{3} ;-\frac{1}{3 \tau}\right) \pm h(3 u \pm \tau ; 3 \tau) e^{\mp 2 \pi i u} .
$$

Hence

$$
\sum_{ \pm} \pm e^{\mp 2 \pi i u} S(3 u \pm \tau ; 3 \tau)=-\frac{e^{\pi i\left(\frac{3 u^{2}}{\tau}+\frac{\tau}{3}\right)}}{\sqrt{-3 i \tau}} \sum_{ \pm} \pm S\left(\frac{u}{\tau} \pm \frac{1}{3} ;-\frac{1}{3 \tau}\right)+\sum_{ \pm} \pm h(3 u \pm \tau ; 3 \tau) e^{\mp 2 \pi i u}
$$


Now we apply Proposition 3.2. to obtain

$$
\begin{aligned}
S\left(\frac{u}{\tau} \pm \frac{1}{3} ;-\frac{1}{3 \tau}\right)= & q_{1}^{-\frac{1}{6}} e^{2 \pi i\left(\frac{u}{\tau} \pm \frac{1}{3}+\frac{1}{2}\right)} S\left(\frac{3 u}{\tau} \pm 1+\frac{1}{\tau}+1 ;-\frac{3}{\tau}\right)+S\left(\frac{3 u}{\tau} \pm 1+0+1 ;-\frac{3}{\tau}\right) \\
& +q_{1}^{-\frac{1}{6}} e^{-2 \pi i\left(\frac{u}{\tau} \pm \frac{1}{3}+\frac{1}{2}\right)} S\left(\frac{3 u}{\tau} \pm 1-\frac{1}{\tau}+1 ;-\frac{3}{\tau}\right) \\
= & q_{1}^{-\frac{1}{6}} e^{2 \pi i\left(\frac{u}{\tau} \pm \frac{1}{3}+\frac{1}{2}\right)} S\left(\frac{3 u}{\tau}+\frac{1}{\tau} ;-\frac{3}{\tau}\right)+q_{1}^{-\frac{1}{6}} e^{-2 \pi i\left(\frac{u}{\tau} \pm \frac{1}{3}+\frac{1}{2}\right)} S\left(\frac{3 u}{\tau}-\frac{1}{\tau} ;-\frac{3}{\tau}\right) \\
& +S\left(\frac{3 u}{\tau} ;-\frac{3}{\tau}\right),
\end{aligned}
$$

where $q_{1}:=e^{-\frac{2 \pi i}{\tau}}$. Consequently,

$$
\sum_{ \pm} \pm S\left(\frac{u}{\tau} \pm \frac{1}{3} ;-\frac{1}{3 \tau}\right)=2 i q_{1}^{-\frac{1}{6}} \sin \left(\pi \frac{5}{3}\right) \sum_{ \pm} \pm e^{ \pm 2 \pi i \frac{u}{\tau}} S\left(\frac{3 u}{\tau} \pm \frac{1}{\tau} ;-\frac{3}{\tau}\right)
$$

Therefore,

$$
\begin{aligned}
\sum_{ \pm} \pm e^{\mp 2 \pi i u} S(3 u \pm \tau ; 3 \tau)= & -\frac{2 i \sin \left(\frac{5 \pi}{3}\right) e^{\pi i\left(9 u^{2}+\tau^{2}\right) /(3 \tau)} q_{1}^{-\frac{1}{6}}}{\sqrt{-3 i \tau}} \sum_{ \pm} \pm e^{ \pm 2 \pi i \frac{u}{\tau}} S\left(\frac{3 u}{\tau} \pm \frac{1}{\tau} ;-\frac{3}{\tau}\right) \\
& +\sum_{ \pm} \pm h(3 u \pm \tau ; 3 \tau) e^{\mp 2 \pi i u}
\end{aligned}
$$

Hence, by (3.8) and

using $\sin (5 \pi / 3)=-\sqrt{3} / 2$ we have

$$
\widehat{A}_{3}(u,-\tau ; \tau)=A_{3}(u,-\tau ; \tau)-\frac{i}{2} e^{2 \pi i u} \vartheta(\tau ; 3 \tau) \cdot \frac{i e^{\pi i \frac{\left(9 u^{2}+\tau^{2}\right)}{3 \tau}} q_{1}^{-\frac{1}{6}}}{\sqrt{-i \tau}} \sum_{ \pm} \pm e^{ \pm 2 \pi i \frac{u}{\tau}} S\left(\frac{3 u}{\tau} \pm \frac{1}{\tau} ;-\frac{3}{\tau}\right)
$$

$$
+\frac{i}{2} e^{2 \pi i u} \vartheta(\tau ; 3 \tau) \sum_{ \pm} \pm h(3 u \pm \tau ; 3 \tau) e^{\mp 2 \pi i u}
$$

Therefore, the right hand side of (3.7) becomes

$$
\begin{aligned}
\tau e^{\pi i\left(-3 u^{2}-2 u \tau\right) / \tau} \widehat{A}_{3}(u,-\tau ; \tau)= & \tau e^{\pi i\left(-3 u^{2}-2 u \tau\right) / \tau} A_{3}(u,-\tau ; \tau) \\
& -\frac{i}{2} \vartheta(\tau ; 3 \tau) \cdot \sqrt{-i \tau} e^{\pi i \frac{\tau}{3}} q_{1}^{-\frac{1}{6}} \sum_{ \pm} \pm e^{ \pm 2 \pi i \frac{u}{\tau}} S\left(\frac{3 u}{\tau} \pm \frac{1}{\tau} ;-\frac{3}{\tau}\right) \\
& +\frac{i}{2} \tau e^{-\frac{3 \pi i u^{2}}{\tau}} \vartheta(\tau ; 3 \tau) \sum_{ \pm} \pm h(3 u \pm \tau ; 3 \tau) e^{\mp 2 \pi i u} .
\end{aligned}
$$

On the other hand

$$
\begin{aligned}
\widehat{A}_{3}\left(\frac{u}{\tau},-1 ;-\frac{1}{\tau}\right)= & \widehat{A}_{3}\left(\frac{u}{\tau}, \frac{1}{\tau}-\left(1+\frac{1}{\tau}\right) ;-\frac{1}{\tau}\right)=e^{-2 \pi i \frac{u}{\tau}} \widehat{A}_{3}\left(\frac{u}{\tau}, \frac{1}{\tau} ;-\frac{1}{\tau}\right) \\
= & e^{-2 \pi i \frac{u}{\tau}} A_{3}\left(\frac{u}{\tau}, \frac{1}{\tau} ;-\frac{1}{\tau}\right)+\frac{i}{2} e^{-2 \pi i \frac{u}{\tau}} \vartheta\left(\frac{1}{\tau} ;-\frac{3}{\tau}\right) S\left(\frac{3 u}{\tau}-\frac{1}{\tau} ;-\frac{3}{\tau}\right) \\
& +\frac{i}{2} e^{2 \pi i \frac{u}{\tau}} \vartheta\left(-\frac{1}{\tau} ;-\frac{3}{\tau}\right) S\left(3 \frac{u}{\tau}+\frac{1}{\tau} ;-\frac{3}{\tau}\right)
\end{aligned}
$$

where we have used Proposition 3.3 (1)-(3) and Proposition 3.1 (1). Hence

$\widehat{A}_{3}\left(\frac{u}{\tau},-1 ;-\frac{1}{\tau}\right)=e^{-2 \pi i \frac{u}{\tau}} A_{3}\left(\frac{u}{\tau}, \frac{1}{\tau} ;-\frac{1}{\tau}\right)+\frac{i}{2} \vartheta\left(\frac{1}{\tau} ;-\frac{3}{\tau}\right) \sum_{ \pm} \pm e^{ \pm 2 \pi i \frac{u}{\tau}} S\left(\frac{3 u}{\tau} \pm \frac{1}{\tau} ;-\frac{3}{\tau}\right)$. 
From Proposition 3.3 (1)-(3) and (5), we have

$$
\begin{aligned}
\vartheta\left(\frac{1}{3} ;-\frac{1}{3 \tau}\right)= & q_{1}^{\frac{1}{6}} e^{2 \pi i(-1)\left(\frac{1}{3}+\frac{1}{2}\right)} \vartheta\left(1+\frac{1}{\tau}+1 ;-\frac{3}{\tau}\right) \\
& +q_{1}^{0} e^{2 \pi i(0)\left(\frac{1}{3}+\frac{1}{2}\right)} \vartheta\left(1+0+1 ;-\frac{3}{\tau}\right) \\
& +q_{1}^{\frac{1}{6}} e^{2 \pi i(1)\left(\frac{1}{3}+\frac{1}{2}\right)} \vartheta\left(1-\frac{1}{\tau}+1 ;-\frac{3}{\tau}\right) \\
= & q_{1}^{\frac{1}{6}} e^{-2 \pi i \frac{5}{6}} \vartheta\left(\frac{1}{\tau} ;-\frac{3}{\tau}\right)+q_{1}^{\frac{1}{6}} e^{2 \pi i \frac{5}{6}} \vartheta\left(-\frac{1}{\tau} ;-\frac{3}{\tau}\right) \\
= & 2 i \sin \left(-\pi \frac{5}{3}\right) q_{1}^{\frac{1}{6}} \vartheta\left(\frac{1}{\tau} ;-\frac{3}{\tau}\right),
\end{aligned}
$$

with $q_{1}:=e^{-2 \pi i \frac{1}{\tau}}$, as above. Thus

$$
\vartheta\left(\frac{1}{\tau} ;-\frac{3}{\tau}\right)=q_{1}^{-\frac{1}{6}} \frac{1}{2 i \sin \left(-\frac{5 \pi}{3}\right)} \vartheta\left(\frac{1}{3} ;-\frac{1}{3 \tau}\right) .
$$

However, by Proposition 3.3 (4) we have

$$
\vartheta\left(\frac{1}{3} ;-\frac{1}{3 \tau}\right)=-i \sqrt{-3 i \tau} e^{\pi i \tau^{2} / 3 \tau} \vartheta(\tau ; 3 \tau)
$$

Thus

$$
\vartheta\left(\frac{1}{\tau} ;-\frac{3}{\tau}\right)=-q_{1}^{-\frac{1}{6}} \frac{\sqrt{-3 i \tau} e^{\pi i \tau^{2} / 3 \tau}}{2 \sin \left(-\frac{5 \pi}{3}\right)} \vartheta(\tau ; 3 \tau)
$$

Hence

$$
\begin{aligned}
\widehat{A}_{3}\left(\frac{u}{\tau},-1 ;-\frac{1}{\tau}\right)= & e^{-2 \pi i \frac{u}{\tau}} A_{3}\left(\frac{u}{\tau}, \frac{1}{\tau} ;-\frac{1}{\tau}\right) \\
& -\frac{i}{2} q_{1}^{-\frac{1}{6}} \frac{\sqrt{-3 i \tau} e^{\pi i \tau^{2} / 3 \tau}}{2 \sin \left(-\frac{5 \pi}{3}\right)} \vartheta(\tau ; 3 \tau) \sum_{ \pm} \pm e^{ \pm 2 \pi i \frac{u}{\tau}} S\left(\frac{3 u}{\tau} \pm \frac{1}{\tau} ;-\frac{3}{\tau}\right) \\
= & e^{-2 \pi i \frac{u}{\tau}} A_{3}\left(\frac{u}{\tau}, \frac{1}{\tau} ;-\frac{1}{\tau}\right) \\
& -\frac{i}{2} q_{1}^{-\frac{1}{6}} \sqrt{-i \tau} e^{\pi i \frac{\tau}{3}} \vartheta(\tau ; 3 \tau) \sum_{ \pm} \pm e^{ \pm 2 \pi i \frac{u}{\tau}} S\left(\frac{3 u}{\tau} \pm \frac{1}{\tau} ;-\frac{3}{\tau}\right)
\end{aligned}
$$

Combining this with (3.7) and (3.10) we have

$$
\begin{aligned}
e^{-2 \pi i \frac{u}{\tau}} A_{3}\left(\frac{u}{\tau}, \frac{1}{\tau} ;-\frac{1}{\tau}\right)= & \tau e^{\frac{\pi i}{\tau}\left(-3 u^{2}-2 u \tau\right)} A_{3}(u,-\tau ; \tau) \\
& +\frac{i}{2} e^{2 \pi i u} \tau e^{\frac{\pi i}{\tau}\left(-3 u^{2}+u \tau\right)} \vartheta(\tau, 3 \tau) \sum_{ \pm} \pm e^{\mp 2 \pi i u} h(3 u \pm \tau ; 3 \tau) .
\end{aligned}
$$

Finally, note that by (1.15), $\vartheta(\tau ; 3 \tau)=-i q^{-\frac{1}{6}} \eta(\tau)$, and by the modular transformation of $\eta$, namely, $\eta(\tau)=\frac{1}{\sqrt{-i \tau}} \eta\left(-\frac{1}{\tau}\right)$, (3.11) yields

$$
\frac{1}{2} \sum_{ \pm} \pm e^{\mp 2 \pi i u} h(3 u \pm \tau ; 3 \tau)=-q^{\frac{1}{6}}\left(\frac{e^{-2 \pi i u}}{\eta(\tau)} A_{3}(u,-\tau ; \tau)-\frac{e^{\frac{3 \pi i u^{2}}{\tau}} \sqrt{-i \tau}}{\tau} \frac{e^{-2 \pi i \frac{u}{\tau}}}{\eta\left(-\frac{1}{\tau}\right)} A_{3}\left(\frac{u}{\tau}, \frac{1}{\tau} ;-\frac{1}{\tau}\right)\right) .
$$

The first part of the theorem now follows from (3.5), namely $\widetilde{R}(u ; \tau)=\frac{e^{-2 \pi i u}}{\eta(\tau)} A_{3}(u,-\tau ; \tau)$. 


\section{Mordell integrals in finite terms}

In this section Theorem 1.4 is proved. This theorem follows from the results of the Folsom, Ono, and the second author [15] and results of Gordon and McIntosh [16].

Define two families of $q$-series by

$$
\begin{aligned}
& g_{2}(x, q):=\sum_{n=0}^{\infty} \frac{q^{\frac{n(n+1)}{2}}(-q ; q)_{n}}{(x ; q)_{n+1}\left(x^{-1} q ; q\right)_{n+1}} \\
& h_{2}(x, q):=\sum_{n=0}^{\infty} \frac{(-1)^{n} q^{n^{2}}\left(q ; q^{2}\right)_{n}}{\left(x q^{2} ; q^{2}\right)_{n}\left(x^{-1} q^{2} ; q^{2}\right)_{n}} .
\end{aligned}
$$

These families are called "universal" mock theta functions by Gordon and McIntosh [16].

Sketch Proof of Theorem 1.4. (A) This follows from the main theorem of [6]. See also [16].

(B) The second identity follows from equation (1) of [35], and the first is in [14]. For $\tau \in \mathbb{H}^{-}$these functions are mock theta functions and are related to the $g_{2}$ and $h_{2}$ universal families introduced by Gordon and McIntosh [16].

(C) We have the following result of Gordon and McIntosh [16]

$$
q^{r(1-r)} g_{2}\left(q^{r}, q\right)-\sqrt{\frac{\pi}{4 \alpha}} \csc (\pi r) h_{2}\left(e^{2 \pi i r}, q_{1}^{2}\right)=-\sqrt{\frac{\alpha}{\pi}} \int_{0}^{\infty} e^{-\alpha w^{2}} \frac{\cosh ((2 r-1) \alpha w)}{\cosh (\alpha w)} d w
$$

where $q=e^{-\alpha}, q_{1}=e^{-\frac{\pi^{2}}{\alpha}}$, and $|q|<1$ in (4.3). Changing variables with $\pi u=\alpha w$ and then setting $x=\frac{i \alpha}{2 \pi}$ and moving the line of integration back to the real axis gives

$$
-\sqrt{\frac{\alpha}{\pi}} \int_{0}^{\infty} e^{-\alpha x^{2}} \frac{\cosh ((2 r-1) \alpha x)}{\cosh (\alpha x)} d x=\frac{1}{\sqrt{2 i x}} \int_{0}^{\infty} e^{\frac{\pi i}{2 x} w^{2}} \frac{\cosh ((2 r-1) \pi w)}{\cosh (\pi w)} d w,
$$

the integral on the right hand side now clearly converges for $r \in(0,1)$.

Moreover, by (2.7) of [15], with $\rho=e^{-2 \pi i \tau}$ we have

$$
G(a, b ; \tau)=-\rho^{\frac{a}{b}\left(1-\frac{a}{b}\right)} g_{2}\left(\rho^{\frac{a}{b}}, \rho\right)
$$

and

$$
h_{2}\left(e^{2 \pi i \frac{a}{b}} ; \rho\right)=\left(1-\zeta_{b}^{-a}\right) \rho^{\frac{1}{8}} H(a, b ; \tau) .
$$

Finally, $H(a, b ;-x)$ and $G(a, b, 1 / 2 x)$ are finite sums for $x \in Q_{a, b}$ by Theorem 1.3 of [15]. Hence (4.3) with $r=a / b$ gives Theorem 1.4.

Proof of Theorem 1.2. Note that

$$
B\left(q^{\frac{1}{2}}\right)=g_{2}\left(q^{\frac{1}{2}} ; q\right)=-q^{-\frac{1}{4}} G(1,2,-\tau)
$$

where the first equality is by definition and the second follows from (4.4) (this is also given in (5.1) of [16]).

To establish the claim we use Theorem 1.4 with

$\int_{0}^{\infty} \frac{e^{-\pi i \frac{k}{2} x^{2}}}{\cosh (\pi x)} d x=\sqrt{\frac{2}{k}} e^{-\frac{\pi i}{4}} \int_{0}^{\infty} \frac{e^{\frac{2 \pi i}{k} x^{2}}}{\cosh (\pi x)} d x=\sqrt{\frac{2}{k}} e^{-\frac{\pi i}{4}} \sum_{n=0}^{\infty} \frac{1}{n !}\left(\frac{2 \pi i}{k}\right)^{n} \int_{0}^{\infty} \frac{x^{2 n}}{\cosh (\pi x)} d x$.

The first equation follows from (1) of [34] or Proposition 1.2 (5) of [42]. The second result follows from Taylor expanding the exponential in the integral and using $\int_{0}^{\infty} \frac{x^{2 n}}{\cosh (\pi x)} d x=\frac{(-1)^{n} E_{2 n}}{2^{2 n+1}}$ (see, for instance, [13]). 


\section{Competing interests}

The authors declare that they have no competing interests.

\section{Authors' contributions}

$\mathrm{BC}$ assisted in the computations, RR carried out the proofs and drafted the manuscript. Both authors read and approved the final manuscript.

\section{Author details}

${ }^{1}$ Department of Electrical Engineering, Stanford University, Stanford, CA 94305, USA. ${ }^{2}$ Department of Mathematics, Stanford University, Stanford, CA 94305, USA.

Received: 2 October 2014 Accepted: 2 October 2014

Published online: 01 July 2015

\section{References}

1. Andrews, GE: Mordell integrals and Ramanujan's "lost" notebook. In: Analytic number theory (Philadelphia, Pa., 1980), Lecture Notes in Math., 899, pp. 10-18. Springer, Berlin-New York, (1981)

2. Bringmann, K, Folsom, A: On the asymptotic behavior of Kac-Wakimoto characters. Proc. Am. Math. Soc. 141(5), 1567-1576 (2012)

3. Bringmann, K, Folsom, A, Rhoades, RC: Partial and mock theta functions as $\boldsymbol{q}$-hypergeometric series. The Ramanujan Journal, special issue "Ramanujan's 125th anniversary special volume". 29(1-3), 295-310 (2012)

4. Bringmann, K, Ono, K: Dyson's ranks and Maass forms. Ann. Math. 171(1), 419-449 (2010)

5. Bringmann, $\mathrm{K}$, Ono, K: The $f(q)$ mock theta function conjecture and partition ranks. Invent. Math. 165(2), 243-266 (2006)

6. Bringmann, K, Ono, K, Rhoades, RC: Eulerian series as modular forms. J. Am. Math. Soc. 21, 1085-1104 (2008)

7. Cheng, MCN: K3 Surfaces, $N=4$ Dyons, and the Mathieu Group $M_{24}$. Commun. Num. Theor. Phys. 4, 623-658 (2010)

8. Cheng, MCN, Duncan, J, Harvey, J: Umbral Moonshine, arXiv Preprint available at arXiv:1204.2779 (http://arxiv.org/ pdf/1204.2779)

9. Dabholkar, A, Murthy, S, Zagier, D: Quantum Black Holes, Wall Crossing, and Mock Modular Forms. arXiv:1208.4074, to appear in Cambridge Monographs in Mathematical Physics, 151 pages

10. Dyson, F: Some guesses in the theory of partitions. Eureka (Cambridge). 8, 10-15 (1944)

11. Eguchi, T, Hikami, K: Superconformal Algebras and Mock Theta Functions 2. Rademacher Expansion for K3 Surfaces. Commun. Number Theory Phys. 3, 531-554 (2009)

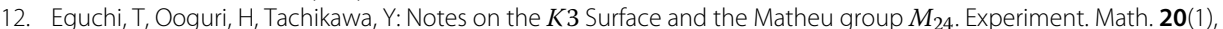
91-96 (2011)

13. Erdelyi, A, Magnus, W, Oberhettinger, F, Tricomi, F: Higher transcendental functions. Vol. III., based on notes left by Harry Bateman, reprint of the 1955 original. Robert E. Krieger Publishing Co., Inc., Melbourne, Fla. (1981). Available at http://apps.nrbook.com/bateman/Vol3.pdf

14. Fine, NJ: Basic hypergeometric series and applications. Math. Surv. Monographs, no. 27, Amer. Math. Soc., Providence (1988). Can be purchased at: http://www.ams.org/bookstore-getitem/item\%3DSURV-27

15. Folsom, A, Ono, K, Rhoades, RC: $q$-series and quantum modular forms. Forum Math. Pi. 1(e2), 1-27 (2013)

16. Gordon, B, Mclntosh, R: A survey of classical mock theta functions. Partitions, $q$-series and modular forms. Dev. Math. 23, 95-144 (2012)

17. Hickerson, D, Mortenson, ER: Hecke-type double sums, Appell-Lerch sums, and mock theta functions (I). Proceedings of the London Mathematical Society. 109(3), no. 2, 382-422 (2014)

18. Hikami, K: Mock (false) theta functions as quantum invariants. Regul. Chaotic Dyn. 10(4), 509-530 (2005)

19. Kronecker, L: Summierung der Gausschen Reihen $\sum_{h=0}^{h=n-1} e^{2 h^{2} \pi i / n}$. J. für die reine und angewandte Mathematik. 105, 267-268 (1889)

20. Kronecker, L: Zur Dastellung von Reihen durch Integrale. Ibid. 105, 345-354 (1889)

21. Kuznetsov, A: Computing the truncated theta function via Mordell integral. Preprint available at http://arxiv.org/abs/ 1306.4081

22. Lawerence, R, Zagier, D: Modular Forms and Quantum Invariants of 3-manifolds. Asian J. Math. 3, $93-107$ (1999)

23. Lerch, M: Bemerkungen zur Theorie der elliptischen Funktionen. Rozpravyceske Akademie cisare Frantiska Josefa pro vedy slovesnost, a umeni (II Cl) Prag. (Bohmisch) I Nr. (24) 1892. Abstract in the Jahrbuch über die Fortschritte der Mathematik. 24, 442-445 (1892)

24. Lerch, M: Beiträge zur Theorie der elliptischen Funktionen, unendlichen Reihen und bestimmten Integrale. Rozpravyceske Akademie cisare Frantiska Josefa pro vedy slovesnost, a umeni (II Cl) Prag. (Bohmisch) II Nr, 23 (1983)

25. Lerch, M: Zur Theorie der Gausschen Summen. Mathematische Annalen. 57, 554-567 (1903)

26. Li, Y, Ngo, H, Rhoades, RC: Renormalization, shadows of mock modular forms, and quantum modular forms. Part II: Mock Theta Functions. arXiv:1311.3044 [math.NT]

27. McIntosh, RJ: Second order mock theta functions. Canad. Math. Bull. 50, 284-290 (2007)

28. Mordell, LJ: The definite integral $\int_{-\infty}^{\infty} e^{a x^{2}+b x} d x /\left(e^{c t}+d\right)$ and the analytic theory of numbers. Acta Math. Stockholm. 61, 323-360 (1933)

29. Mordell, LJ: The value of the definite integral $\int_{-\infty}^{\infty} e^{a t^{2}+b t} d t /\left(e^{c t}+d\right)$. Quarterly J. Math. 68, 329-342 (1920)

30. Mortenson, ET: On the dual nature of partial theta functions and Appell-Lerch sums. Advances in Mathematics. 264 236-260 (2014)

31. Ono, K: Unearthing the visions of a master: harmonic Maass forms and number theory. In: Proc. 2008 Harvard-MIT Current Developments in Mathematics Conf, pp. 347-454, Somerville, Ma, (2009)

32. Pirjol, D: The logistic-normal integral and its generalizations. J. Comput. Appl. Math. 237, 460-469 (2013)

33. Rademacher, H: A Convergent Series for the Partition Function $p(n)$. Proc. Nat. Acad. Sci. 23(2), 78-84

34. Ramanujan, S: Some Definite Integrals connected with Gauss's sums. Messanger Math. 44, 75-85 (1915) 
35. Rogers, L: On two theorems of combinatory analysis and some allied identities. Proc. London Math. Soc. 16, 315-336 (1917)

36. Rhoades, RC: A unified approach to partial and mock theta functions. To appear in Mathematical Research Letters. Preprint available at http://math.stanford.edu/ rhoades/FILES/expansion.pdf

37. Siegel, CL: Über Riemanns Nachlass zur analytischen Zahlentheorie. Quellen und Studien zur Geschichte de Mathematik, Astromie und Physik, 2, 45-80 (1933)

38. Zagier, D: Vassiliev invariants and a strange identity related to the Dedekind eta-function. Topology. 40(5), 945-960 (2001)

39. Zagier, D: Ramanujan's mock theta functions and their applications (after Zwegers and Ono-Bringmann). Séminaire Bourbaki. Vol. 2007/2008. Astérisque No. 326 (2009), Exp. No. 986, vii-viii, 143-164 (2010)

40. Zagier, D: Quantum modular forms. In Quanta of Maths: Conference in honor of Alain Connes, Clay Math. Proc. Amer. Math. Soc., Providence. 11, 659-675 (2010)

41. Zwegers, S: Mock theta functions and real analytic modular forms. Contemporary Math. 291, 269-277 (2001)

42. Zwegers, S: Mock Theta Functions. Thesis, Utecht (2002)

43. Zwegers, S: Multivariable Appell Functions. Preprint available at http://citeseerx.ist.psu.edu/viewdoc/download? doi=10.1.1.164.6121\&rep=rep1\&type=pdf

\section{Submit your manuscript to a SpringerOpen ${ }^{\odot}$ journal and benefit from:}

- Convenient online submission

$\checkmark$ Rigorous peer review

- Immediate publication on acceptance

- Open access: articles freely available online

- High visibility within the field

- Retaining the copyright to your article

Submit your next manuscript at $\boldsymbol{\triangleright}$ springeropen.com 